\title{
Infiltración de un gel biocompatible para el tratamiento de las exóstosis interdigitales. Experimentación en cadáver
}

\author{
Infiltration of a biocompatible gel for the treatment of interdigital \\ exostoses: A cadaveric study
}

\author{
Enrique Giralt de Veciana ${ }^{1}$, Elena de Planell Mas $^{2}$, Elvira M $^{a}$ Bonilla Toyos ${ }^{3}$, \\ Virginia Novel Martí ${ }^{4}$, Consuelo Truchero Coma ${ }^{5}$, M $^{\text {a }}$ Cristina Manzanares Céspedes ${ }^{6}$ \\ ${ }^{1}$ Profesor Titular. Departamento de Podología. Universidad de Barcelona \\ egiralt@ub.edu \\ ${ }^{2}$ Profesor Colaborador. Departamento de Podología. Universidad de Barcelona \\ elenaplanell@ub.edu \\ ${ }^{3}$ Profesor Máster Oficial Podología Quirúrgica. Departamento de Podología. Universidad de Barcelona \\ elbonilla@inicia.es \\ ${ }^{4}$ Profesor Titular. Departamento de Podología. Universidad de Barcelona \\ vnovel@ub.edu \\ ${ }^{5}$ Profesor Máster Oficial Podología Quirúrgica. Departamento de Podología. Universidad de Barcelona \\ trucherocoma@hotmail.com \\ ${ }^{6}$ Profesor Titular. Departamento de Patología y Terapia Experimental \\ mcmanzanares@ub.edu
}

Correspondencia:

Dr. Enrique Giralt de Veciana

Univesidad de Barcelona. Departamento de Podología

Pabellon de Gobierno.

C/ Feixa Llarga s/n

08907 L'Hospitalet de Llobregat

Barcelona (Spain)

E-mail: egiralt@ub.edu

Fecha de recepción: 24 enero de 2012

Fecha de aceptación: 22 mayo de 2012

Los autores declaran no tener ningún tipo de interés económico o comercial.

\section{RESUMEN}

Antecedentes. La exóstosis interdigital es una patología común que se presenta con frecuencia en las consultas de podología. A pesar del alto grado de incidencia es una entidad clínica muy poco estudiada. En la actualidad, los tratamientos propuestos para resolver esta patología son el tratamiento conservador mediante ortesis de silicona y el tratamiento quirúrgico consistente en la resección de la exostosis. Consideramos necesario otra alternativa de tratamiento que mejore la actividad diaria de los pacientes que presenten esta patología.

Material y Métodos. Se procedió a la infiltración de un gel biocompatible en cadáver para simular de la forma más idónea posible la posterior aplicación clínica en pacientes. Se seleccionaron 10 pies de cadáver fresco, no formolado, con presencia de exostois a los que se realizó la infiltración del gel biocompatible siguiendo las instrucciones del fabricante.

Resultados. Se observaron los siguientes aspectos: relación cantidad de producto/dimensiones obtenidas, edad de los especímenes, sexo, localización exostosis, cantidad de producto infiltrado, tipo de aguja, forma de infiltración, facilidad de la infiltración y distribución del producto.

Conclusiones. La valoración de la técnica es positiva en el 100\% de los casos, demostrando que el producto permanece in situ en la localización prevista, lo que nos hace concluir que puede ser un tratamiento válido para la exostosis interdigital.

Palabras clave: Exostosis Interdigital; Gel Poliacrilamida; Infiltración. 


\section{ABSTRACT}

Precedents. Interdigital exostosis is a common pathology in podiatric surgery. Despite the rates of incidence, little has been researched on this clinical entity. Currently proposed treatments are: conservative treatment with silicone orthosis, and surgical treatment based on resection of exostosis. An alternative treatment to improve daily activity of patients with this pathology has been considered necessary.

Material and Methods. Bio compatible gel was injected in a dead body in order to experiment and simulate the subsequent clinical application in patients. We selected 10 feet of fresh dead body, non-preserved in formaldehyde, with presence of exostosis, to which the bio compatible gel was injected following the established protocol and instructions provided by the manufacturer.

Results. Aspects observed: relationship quantity of product / dimensions obtained, age of specimen, gender, location of exostosis, quantity of product injected, type of needle, injection procedure, ease of injection and allocation of product.

Conclusion. The method is assessed as positive in $100 \%$ of studied cases, as the product remains in the predicted area, therefore we conclude that it is a suitable treatment for the interdigital exostosis.

Key words: Interdigital exostosis; Polyacrylamide Gel; Injection.

Sumario: 1. Introducción, 2. Material y Métodos, 3. Resultados, 4. Discusión, 5. Anexo, Bibliografía.

Referencia normalizada: Giralt de Veciana, E., de Planell Mas, E., Bonilla Toyos, EM., Novel Martí, V., Truchero Coma, C., Manzanares Céspedes, MC. Infiltración de un gel biocompatible para el tratamiento de las exóstosis interdigitales. Experimentación en cadáver. Rev. Int. Cienc. Podol. 2012; 6(2): 95-110. 


\section{INTRODUCCIÓN}

La exostosis, según define Canella ${ }^{4}$ es una hipertrofia parcial circunscrita a la superficie de un hueso que puede aparecer como una lesión aislada o de forma múltiple, constituyendo entonces la denominada Exóstosis Múltiple Hereditaria.

La exóstosis presenta un recubrimiento a modo de cofia de tejido fibrocartilaginoso, como la describe De Palma ${ }^{15}$, con características histológicas semejantes a las de un callo de fractura, pudiendo aparecer en varias localizaciones en el pie, siendo las más frecuentes la subungueal y la interdigital.

Tanto Landon ${ }^{21}$ como Canella ${ }^{4}$ coinciden en que la edad de presentación más frecuente es la segunda década de la vida, independientemente de que pueda aparecer asimismo en población infantil o en la población adulta o geriátrica, ya que la edad de presentación varía desde los 4 hasta los 70 años.

Nuestra experiencia clínica nos ha puesto de manifiesto que la exóstosis se observa más frecuentemente en el sexo femenino. Según las series publicadas por Evison y Price ${ }^{17}$, la exostosis es en efecto más común en mujeres que en hombres, lo que estos autores atribuyen al uso del calzado más apretado y estrecho que ellas usan. Las exostosis, usualmente, son solitarias $\mathrm{y}$ unilaterales.

Respecto a la causa de la exóstosis, se han postulado varias teorías, como por ejemplo considerarlas como formas de la exóstosis múltiple hereditaria o como una malformación originada en restos de cartílago situados bajo el periostio de la falange ${ }^{23}$. También se ha constatado en la bibliografía que la exóstosis no es un tumor verdadero sino excrecencias constituidas por tejido óseo normal y cartílago calcificado. La mayoría de las descripciones de la exóstosis la presentan como una condición benigna más bien de tipo reactivo que neoplásico. Aunque frecuentemente se menciona en la literatura el traumatismo como posible causa, no existe evidencia clara que este sea el factor desencadenante. Tampoco la inflamación o infecciones explican la etiología $a^{16,28,31}$.

Actualmente la causa más aceptada parece ser la traumática. En este sentido se hace referencia a un traumatismo directo o bien a un microtraumatismo repetido, el cual provocaría una irritación permanente del periostio, que iría causando lentamente la formación de la exóstosis.

Por otro lado Butterworth ${ }^{3}$ considera como factor primario un desequilibrio biomecánico como por ejemplo la inestabilidad del arco longitudinal del pie.

En este mismo sentido, De Palma et al ${ }^{15}$ refieren que, aunque la relación entre este tipo de lesión y la actividad diaria no esta todavía muy clara, la mayoría de los pacientes de su estudio realizaban una actividad diaria intensa, por lo que el microtrauma debe de ser un factor a tener en cuenta.

La exóstosis por tanto, parece ser producto de una irritación crónica de la falange que puede originarse por factores causales diversos. Ésta se debe tener en cuenta para realizar el tratamiento, ya que se debe identificar y corregir el factor causal de ésta para evitar complicaciones.

La sintomatología más frecuente en las exostosis es un dolor selectivo a la presión directa en la zona de localización de ésta. Dicho dolor es punzante y agudo, lo que limita la deambulación. Además, puede acompañarse de inflamación, infección, formación de helomas e incluso, en casos severos, de ulceración ${ }^{14,10,11,13}$.

En ocasiones se aprecia una deformidad cutánea externa debida a la prominencia de la exóstosis. Sin embargo, la manifestación clínica más frecuente de las exóstosis digitales es la formación de un heloma, definido como una "queratopatía con núcleo que corresponde a una presión directa o intermitente de la piel sobre un punto óseo"1. En el caso de las exóstosis digitales, la piel es presionada por dos estructuras densas: una es la exostosis ósea que constituye el elemento constante y el dedo lateral o el calzado en el caso de exóstosis interdigitales o en dorso de dedo respectivamente ${ }^{27}$ constituye el elemento que, de forma intermitente, ejerce presión sobre la piel y el hueso subyacente.

La experiencia clínica nos ha venido demostrando que en las exostosis de conformación puntiaguda se produce un dolor punzante $y$, un dolor tórpido es el más frecuentemente referido en las exostosis de conformación roma. Con frecuencia, sin embargo, se realizan exploraciones radiológicas en las cuales se puede 
evidenciar la presencia de exóstosis totalmente asintomáticas.

Los estudios histológicos muestran que la lesión ósea de la exostosis se compone de hueso esponjoso maduro sobre el que se dispone una capa de fibrocartílago proliferante ${ }^{26}$ que, como indicó Dahlin ${ }^{12}$ en 1980, reproduce el aspecto histológico de un callo de fractura en sus diversos grados de evolución ${ }^{18}$.

Nuestra experiencia clínica nos muestra que la imagen histológica descrita concuerda con el aspecto quirúrgico. Se observa un grado variable de hiperplasia epitelial, hiperqueratosis y acantosis irregulares en las lesiones secas, bajo las que existe un tejido fibroso denso que, en la profundidad se transforma en tejido fibrocartilaginoso con focos irregulares de osificación, con espículas óseas que se confunden con los tejidos óseos de la falange.

En consecuencia, la mayoría de las exóstosis representa una forma osificante de metaplasia fibrocartilaginosa ${ }^{24}$, que aparece como respuesta a un estímulo crónico cuya naturaleza exacta y su causa no están definidas.

También ha de señalarse el hecho de que el crecimiento de la exóstosis no sea excesivo: los casos más grandes descritos en la literatura son de unos $20 \mathrm{~mm}$ de volumen ${ }^{22}$, lo que se atribuye a que el cese del crecimiento activo se debe al cambio de tejido fibrocartilaginoso a uno fibroso colagenizado que cubre la exóstosis.

El diagnóstico clínico es corroborado por el estudio radiográfico, y se recomienda realizar una radiografía amplificada del dedo afectado en proyecciones anteroposterior y lateral. Una vez bien establecida la lesión se observa una excrecencia de hueso de estructura reticular o esponjosa $a^{6,18}$.

Las exóstosis suelen tener forma de pico, es decir, una base ancha sin corteza bien definida, que se va estrechando a medida que aumenta la altura. Al tratarse de hueso trabecular, la imagen radiográfica, tendrá densidad ósea. Además, se observan las trabéculas óseas en toda la extensión de la exóstosis. No existe diferenciación perióstica, ya que no se aprecia un límite radiológico entre la falange y la exostosis. La exóstosis es de crecimiento excéntrico, y no deforma la cortical del hueso, así como tampoco invade el interior de la cavidad medular ${ }^{20,19,25}$.

No se ha documentado la evolución maligna de las exóstosis a condrosarcoma. Por ello es importante diferenciar la exóstosis de otros tumores de las falanges ${ }^{29}$, dada las similitudes de la exóstosis con el osteocondroma y con el encondroma. Aunque algunos autores consideran a la exóstosis como un osteocodroma, existen claras diferencias entre ellos: los osteocondromas, a diferencia de la exóstosis, se observan más frecuentemente en el sexo masculino; en la exóstosis la cubierta cartilaginosa es de tipo fibroso mientras que en el osteocondroma es de tipo hialino y, más importante, la exóstosis se observa lejos y fuera de la línea epifisaria sin conectarse con ella ${ }^{17}$.

El encondroma ${ }^{34}$ es un crecimiento cartilaginoso excesivo dentro de la cavidad medular del hueso, que adelgaza y distiende la corteza favoreciendo la fractura del mismo. Puede crecer solitario en la falange distal y presentarse clínicamente como una paroniquia ${ }^{29}$. Su imagen, de aspecto quístico radiolúcido en la cavidad medular de la falange, es característica y muy diferente a la de la exóstosis.

Pese a la prevalencia y la revelancia clínica que tiene esta patología para la calidad de vida del paciente, el arsenal terapéutico del que se dispone para un tratamiento curativo no sintomático es limitado.

El tratamiento sintomático está representado por la ortesis de silicona con el objetivo de eliminar el heloma y el dolor que causa, aplicando un tratamiento externo que alivia la presión de los tejidos dérmicos sobre la exostosis y viceversa.

El tratamiento curativo está representado por la cirugía, que se lleva a cabo con el objetivo de eliminar de forma definitiva la exostosis y de esta manera erradicar el heloma y el dolor al suprimir su causa primaria.

La búsqueda de un tratamiento exento de los efectos secundarios de los tratamientos clásicos, con un procedimiento alternativo empleando la técnica de un implante subcutáneo en las zonas de queratosis podal, con el uso de un fluido de densidad variable, que actuaría como amortiguador entre la exostosis y el tejido dérmico, eliminando la presión de la exostosis sobre la dermis y evitando la producción de helomas, nos ha llevado a este estudio.

Creemos que se puede mediante el relleno de Gel de Policrilamida crear una interfase de características parecidas al tejido adiposo de la 
zona alrededor de la exostosis y sobre la misma, amortiguando o acolchando la zona, logrando de esta forma la anulación de una de las dos presiones densas que provoca la formación del heloma.

Al no existir ningún estudio previo de su utilización en esta patología, y antes de su utilización en pacientes, nos proponemos como objetivo principal de este estudio, la experimentación y valoración de la técnica de infiltración en cadáver.

\section{MATERIAL Y MÉTODOS}

\subsection{Planteamiento del estudio}

Como hemos descrito anteriormente en la actualidad como únicos tratamientos se plantea la aplicación de una ortesis de silicona aisladora de la presión, para amortiguar el proceso o bien la realización de una intervención quirúrgica con el objetivo de eliminar la exostosis de forma definitiva.

Ambos tratamientos en algunas ocasiones son rechazados por los pacientes por diversas causas entre las cuales podemos citar: molestia de la colocación diaria de la silicona digital, modificación del calzado utilizado para la adaptación de la silicona, intolerancia a la silicona: eczema o alergias, temor o no aceptación del proceso quirúrgico, rechazo al tratamiento por comentarios negativos de otros pacientes.

La necesidad de establecer tratamientos alternativos nos ha impulsado a estudiar protocolos y productos que pudieran utilizarse como una barrera mecánica entre los tejidos dérmicos y los tejidos óseos de las falanges del pie.

Materiales de uso frecuente en otras especialidades quirúrgicas, como el colágeno, ácido hialurónico o toxina botulínica, no resultaron idóneos tras su estudio teórico, debido a su facilidad para metabolizarse, por lo que no constituían una barrera mecánica estable entre las estructuras anatómicas mencionadas.

\section{A) Implantes subcutáneos como nuevo tratamiento}

El primero de dichos estudios fue iniciado por el Dr. S. W. Balkin (DPM) en California (EEUU) en 1966, y posteriormente modificado en 1975, mediante la implantación subcutánea de una inyección de fluido inyectable Podosil ${ }^{\circledR}$ (Copolymethydiphensylsiloxane) en queratosis del pie, realizando a nivel digital una infiltración de como máximo $0,10 \mathrm{cc}$.

En nuestra experiencia y basándonos en los estudios del Dr. Balkin iniciamos en España en la Clínica Podológica de la Universidad de Barcelona, un proyecto por los investigadores Virginia Novel i Marti, Enrique Giralt de Veciana y Antonio Viladot Voegeli en 1996, que obtuvieron los permisos administrativos para la realización del protocolo de investigación completo.

Debido a la problemática a nivel sanitario y legal que la implantación de silicona en el organismo provocó a nivel mundial y sobre todo en Estados Unidos (el Podosil fue elaborado por Biodermis- Corporation de las Vegas); ocasionó la paralización del proyecto de investgación en España y la finalización de la aplicación de dicha terapia por parte del Dr. Balkin.

Los resultados iniciales del procedimiento quirúrgico consistente en la inyección de un material amortiguador a nivel de la exostosis y la dificultad de formación del heloma y sus consecuencias nos empujaron a experimentar un material alternativo. Este material debe ser estable en el tiempo, no reabsorvible y permitir la integración en la estructura dérmica, para evitar intolerancias o efectos secundarios.

Por ello hemos seleccionado un material inyectable, ya utilizado en otras zonas del organismo y que cumple las características de integración en la estructura dérmica y es estable en el tiempo. Este material viene siendo utilizado en procedimientos quirúrgicos por cirujanos plásticos y estéticos con resultados muy satisfactorios desde su comercialización ${ }^{32,33}$.

Se trata del GEL DE POLICRYLAMIDA (PAAG) comercializado como AQUAMID ${ }^{\circledR}$ Reconstruction [Contura Industries, Soeborg, (Dinamarca)]

B) PAAG: Características y mecanismo de acción

Aquamid ${ }^{\circledR}$ Reconstruction o PAAG es un hidrogel inyectable compuesto del $97,5 \%$ de agua y el $2,5 \%$ de poliacrilamida reticulada patentada. El agua se une a una forma de polímero de poliacrilamida patentada, de composición homogénea, sin micropartículas. 
El PAAG al incorporarse al tejido orgánico receptor intercambia agua, sales y moléculas orgánicas con el mismo. Lo que comporta una alta garantía de biocompatibilidad y excelentes resultados estéticos ${ }^{30}$.

Debido a la amplia experiencia disponible ${ }^{35,7,8,9}$ sobre la utilización quirúrgica del producto diversos estudios han demostrado unas características del PAAG muy positivas:

- No es degradable.

- No alérgico, atóxico.

- Homogéneo sin liberación de partículas

- Intercambia agua, sales y moléculas orgánicas con el tejido receptor

- Se almacena sin refrigeración.

Los mecanismos de acción descritos en el organismo ${ }^{35,7,8,9}$ son :

- Aumento del volumen de la zona infiltrada sin reacciones a cuerpos extraños.

- Total integración y vascularización.

- Satisfacción duradera de los pacientes

Las poliacrilamidas han sido utilizadas desde hace mucho tiempo en productos médicos y en la purificación de agua y alimentos ${ }^{5}$ como:

- Producción de lentes de contacto blandas y lentes intraoculares.

- Ingrediente para geloesferas microencapsuladas utilizadas en medicamentos.

- Productos para envasar alimentos.

- Agente floculante en la sedimentación y purificación del agua.

- Clarificación de jugos de azúcar de remolacha y de caña.

Christensen ${ }^{9}$ describe el mecanismo de acción de PAAG en el tejido subcutáneo:

- El efecto de relleno se debe exclusivamente al volumen inyectado.

- El efecto de relleno no depende de la reacción intencionada de un cuerpo extraño a microparticulas que actúan como irritantes. Por lo tanto no genera endurecimiento ni fibrosis del tejido.

- El PAAG se integra totalmente en el tejido a través de una red fibrosa fina. Con el tiempo el hidrogel se vasculariza, lo que permite el acceso al sistema inmune.

- El PAAG está en intercambio continuo de agua con el tejido adyacente, lo que impide la encapsulación del implante.
El PAAG sólo debe inyectarse por vía subcutánea y no en la dermis, se debe realizar mediante la técnica de inyección retrógrada en líneas múltiples.

El mecanismo mediante el que se integra PAAG al tejido se produce por la formación de una red en el hidrogel, los macrófagos son los primeros elementos celulares que ingresan en dicha red de hidrogel y con el tiempo los fibroblastos forman hilos de tejidos conectivos dentro del hidrogel.

A los 14 meses se puede comprobar mediante biopsia la formación de una red fina de hilos del tejido conectivo receptor y mediante biopsia a los 4 años de su infiltración se ha podido demostrar la perfecta integración del hidrogel PAAG en el tejido receptor, mediante una fina red de hilos en el tejido conectivo, pudiendo estar rodeado de fibras musculares, células adiposas y tejido conectivo, tal y como hemos descrito anteriormente.

El PAAG ya ha sido utilizado y experimentado ampliamente en otras zonas del organismo con fines plásticos y/o estéticos, con gran satisfacción por parte de los pacientes ${ }^{2,32}$.

\section{Técnica de infiltración de PAAG}

Utilizamos el siguiente protocolo de manejo:

Se retira el protector Tip Cap, girándolo en la misma dirección que se usa para fijar la aguja. Se conecta la aguja a la base del Luer lock y se verifica que la aguja esté montada correctamente. [Si la aguja estuviera obstruida, no debemos aumentar la presión sobre el émbolo, sino la inyección para reemplazar la aguja]. Se inyecta la cantidad necesaria del PAAG subcutáneamente de forma retrógrada, como un abanico, inyectando el gel a medida que se va retirando la aguja. Habrá que evitar todo exceso de corrección por inyección de exceso de gel.

Una vez administrado el volumen necesario de gel, aflojamos la presión sobre el émbolo antes de proceder a retirar la aguja. Tras la inyección, realizamos un ligero masaje para garantizar una distribución uniforma del gel.

\section{Experimentación en cadáveres}

Previa actuación sobre tejido in vivo, es decir sobre pacientes, realizamos una experimentación sobre cadáver fresco, no formolado, para simular de la forma más idónea posible la pos- 
terior aplicación clínica en pacientes del método para comprobar, tanto el método quirúrgico como el manejo del material y su disposición en las estructuras anatómicas del pie-.

Partiendo del conocimiento del producto y de la forma de aplicación en otras zonas del organismo, se planteó su aplicación en el pie y concretamente en los dedos sobre la superficie de una exostosis, tal como hemos programado en nuestro protocolo.

\section{A. Metodología}

- Hemos seleccionado diez pies de cadáver con presencia de exostosis, previamente identificada por fluoroscopia y posterior radiografía con placa radiográfica oclusal (Fig. 1).

- Identificamos cada uno de los pies para registrar los datos correspondientes a cada procedimiento experimental (Anexo). Asimismo, se procedió al registro fotográfico

- Se prepararon los pies de cadáver con el mismo protocolo de aseptización aplicado a los pacientes in vivo. Se realizó el protocolo quirúrgico de lavado con jabón aseptizante, enjuague con alcohol de $97^{\circ}$ y aplicación de Clorhexidina.

- La identificación del PAAG en la posterior disección de los dedos de cada espécimen podía verse dificultada, debido

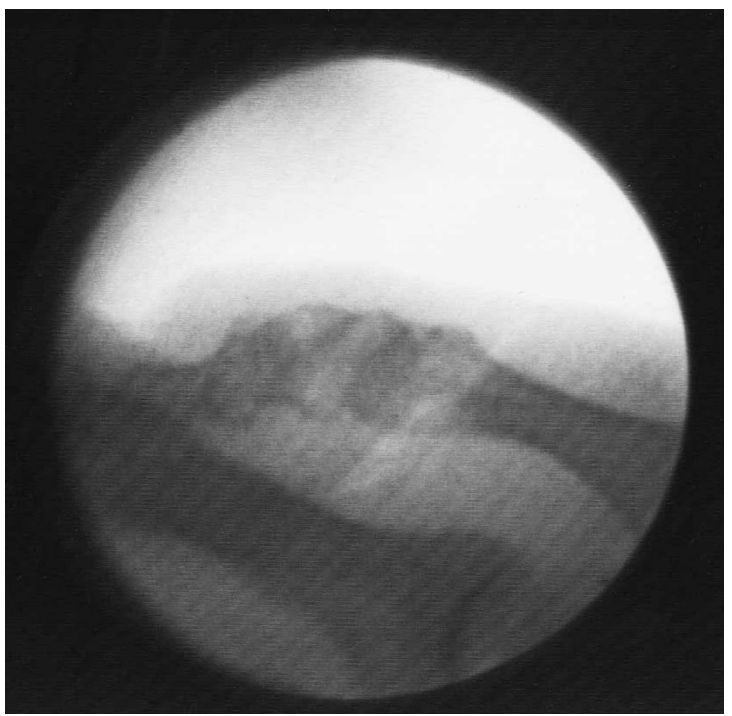

Fig. 1. Fluoroscopia y localización exostosis. a la transparencia del gel, por lo que se procedió a la tinción del mismo con azul de metileno al 15\%, con el fin de localizarlo (Fig. 2). Un punto importante del estudio preliminar consistía en comprobar que en los especimenes no se producía una diseminación y/o infiltración del gel a tejidos adyacentes, con la consiguiente alteración de los mismos.

- Se localiza el punto de punción colocando el bisel de la aguja sobre el punto central de la exóstosis localizada mediante palpación.

- Se punciona en el dedo a nivel distal de la exóstosis con una aguja de $25 / 5$ y otra de 25/6 para registrar en cada calibre la facilidad de infiltración del producto en él y de esta forma determinar cual será la que nos dará mayores ventajas en pacientes, para lograr la mejor distribución del producto con el menor trauma y dolor para éste.

- El producto se distribuyó siguiendo las recomendaciones del fabricante, en forma de abanico, con el vértice de dicho ángulo en el punto de punción, realizándose las líneas convergentes necesarias en cada caso. (Fig. 3-4). Finalizado el protocolo, se realizó una nueva fluoroscopia para observar si la distribución del material había sido efectiva.

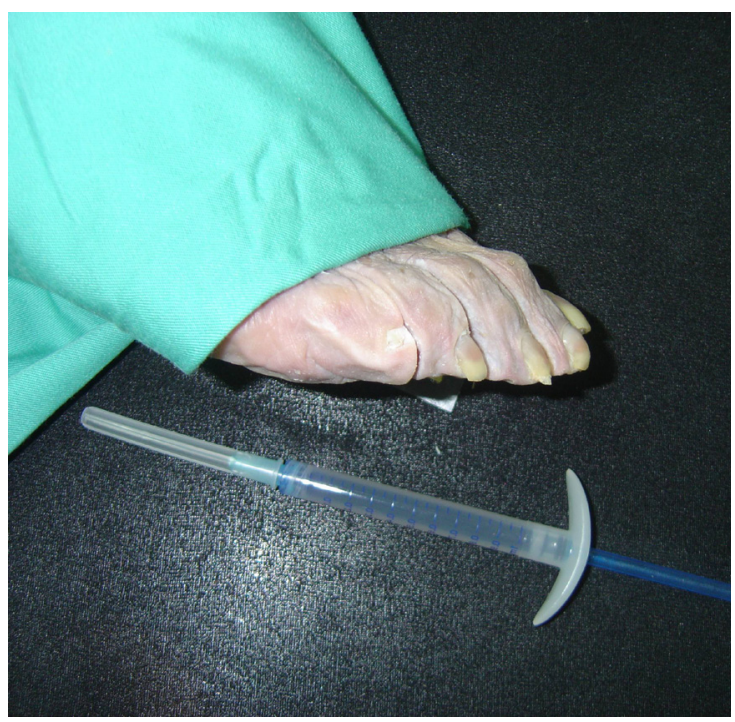

Fig. 2. Preparación del espécimen y tintado del PAAG. 
- Por la tinción del PAAG y la transparencia del tejido dérmico en la localización subcutánea se pudo observar la posición del gel. Se procedió a realizar con bario un círculo alrededor de la tinción azulada seguida de la realización de radiografías de control para poder comprobar si se había inyectado material en la zona de la exostósis y si abarcaba la totalidad de la misma. (Fig. 5-6)

- Seguidamente en la mitad de los especímenes se realizó la disección por planos de todas las estructuras anatómicas del dedo para observar "in situ" la distribución del material, confirmando la correcta realización de la técnica de apli-

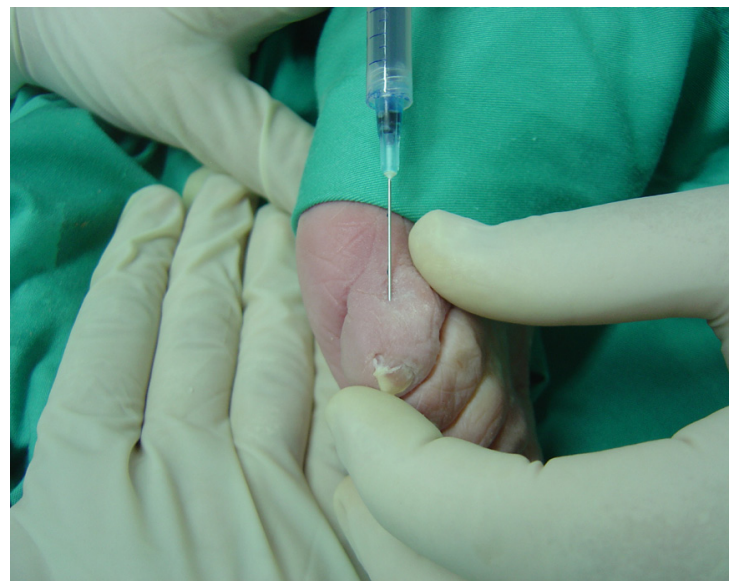

Fig. 3. Punción e infiltración del producto.

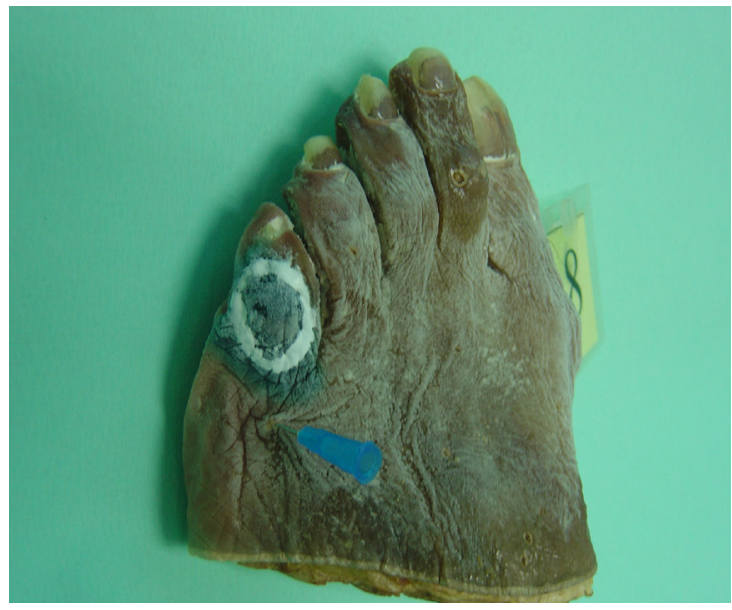

Fig. 5. Marcado externo con bario del contorno de la lesión. cación. Se pudo observar que el PAAG se encontraba en el tejido subcutáneo, no invadiendo, ni diseminándose a otras capas tisulares y sobre todo no infiltraba a la cápsula articular y por tanto no afectaba en absoluto a ninguno de los componente articulares, $n i$ al tejido cartilaginoso, ni al óseo (Fig. 7-8)

- En el resto de especímenes se realizó una sección sagital del dedo operado, para intentar comprobar la disposición del biomaterial inyectado sin alterar las relaciones anatómicas. (Fig. 9) Observándose igualmente la no afectación, ni infiltración en otras capas tisulares de las estructuras anatómicas, aparte del tejido

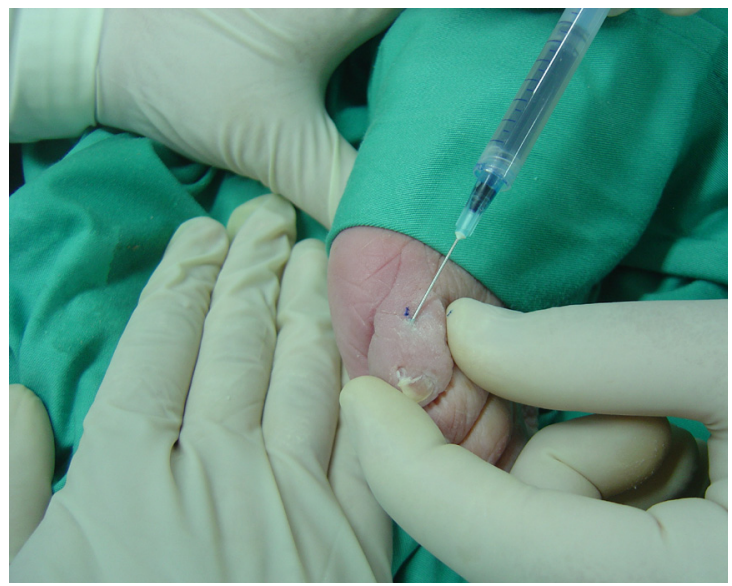

Fig. 4. Lateralización de la aguja para infiltrar en abanico.

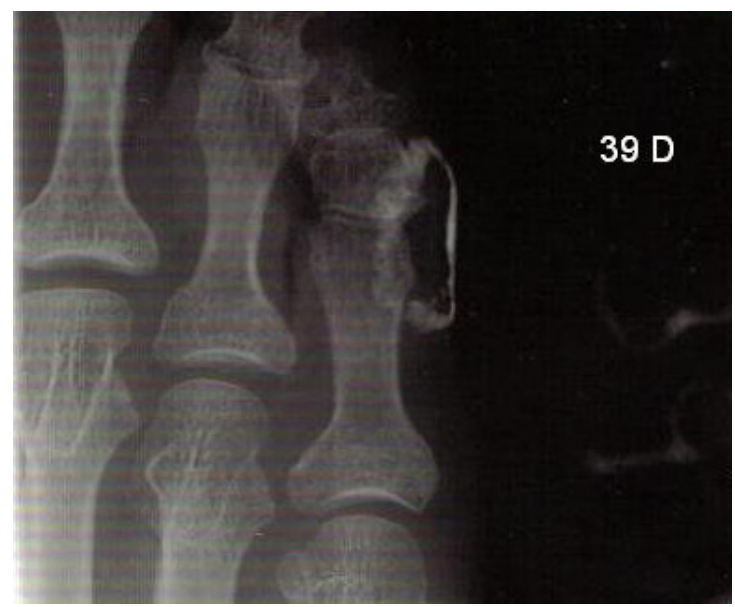

Fig. 6. Radiografía de comprobación del marcaje. 
celular subcutáneo, tal como había sido descrito por la literatura (Fig. 10). Comprobándose igualmente el grosor del producto localizado sobre la exóstosis.

Todos los datos seleccionados en los especimenes y observados en ellos fueron trasladados a la hoja de control de los especímenes, (Anexo) donde se registraron para su posterior análisis:

- Datos facilitados por la sala de disección del Campus de Bellvitge de la Universidad de Barcelona: identificación según el registro de Sala, sexo y edad.

- Datos de la zona a explorar: Localización topográfica de la lesión, medio con que se detecto la exostosis.

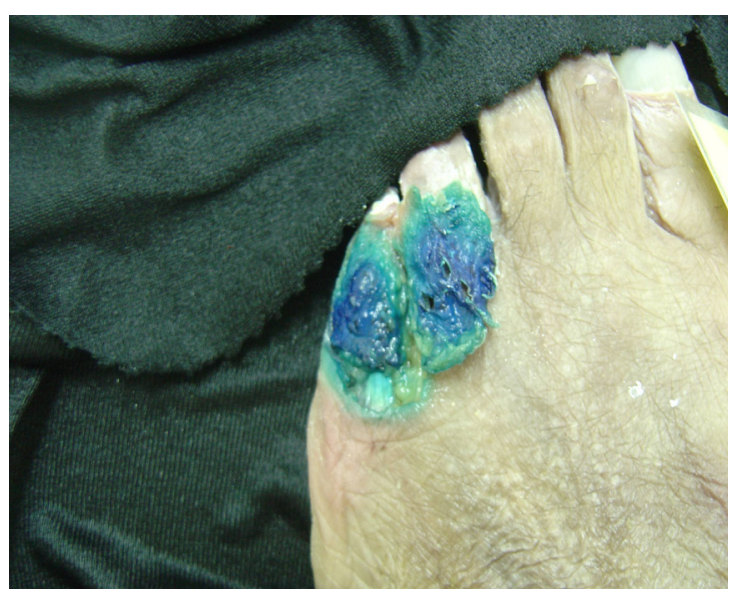

Fig. 7. Disección del dedo y observación de la distribución del producto.

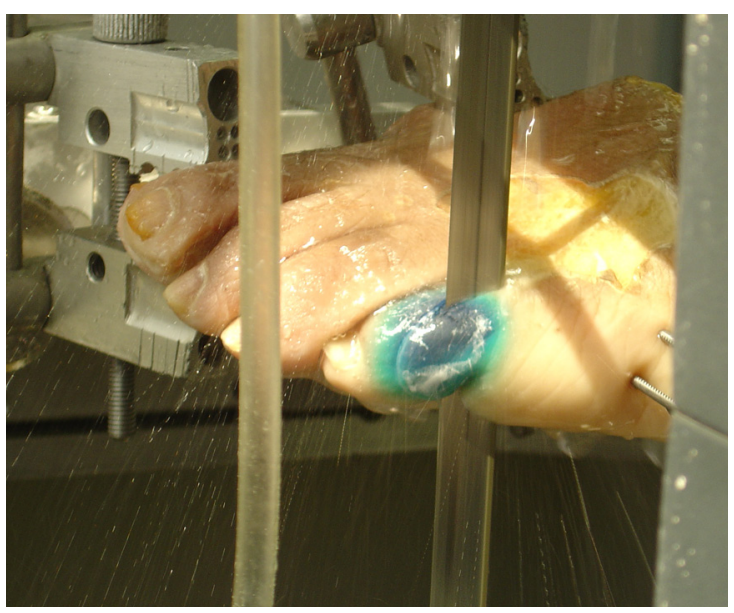

Fig. 9. Sección sagital del dedo.
- Datos referentes a la aplicación mediante infiltración de PAAG en el espécimen: Cantidad de producto infiltrado; tipo de aguja empleado (calibre y longitud), forma de aplicación de la técnica; facilidad de infiltración del producto, observación de la distribución que como ya se ha explicado se realizó en el 50\% de los especímenes.

- Valoración integral de la técnica definiendo los resultados planteados al iniciar el ensayo, de lograr un material que rellene y aísle la exostosis de posibles presiones o bien una valoración negativa de la misma por no haber logrado los objetivos del experimento.

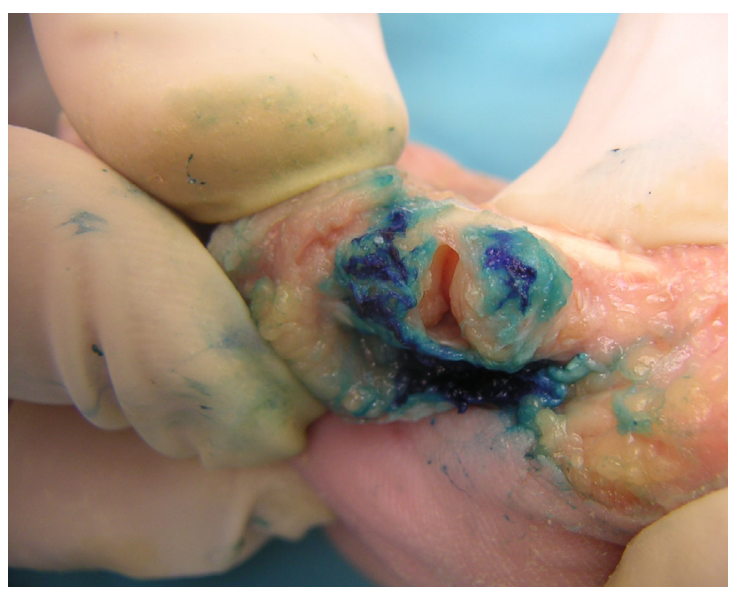

Fig. 8. Observación de la distribución del producto tras la disección de la articulación.

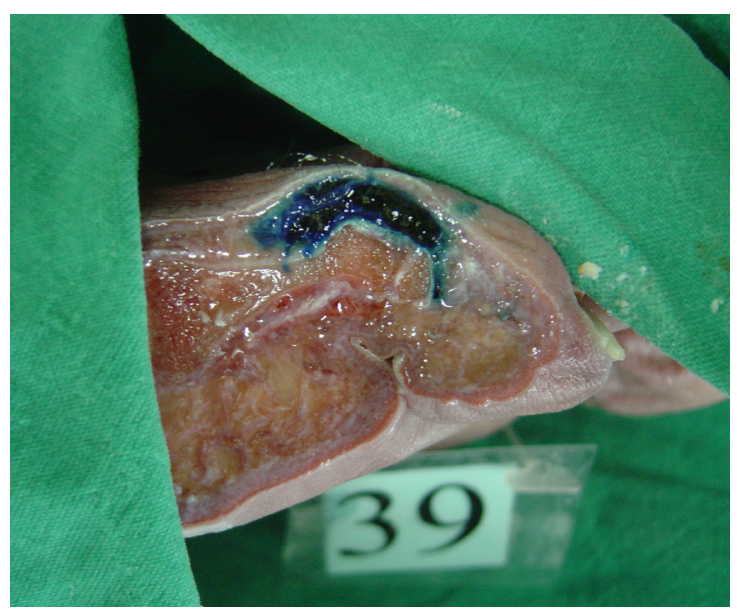

Fig. 10. Observación de la distribución del producto en el dedo seccionado. 


\section{RESULTADOS}

Relación cantidad de producto/dimensiones obtenidas

A fin de establecer el método quirúrgico definitivo, dos observadores -el operador y un observador experto- midieron y registraron la cantidad de PAAG inyectada (en cc) y midieron las dimensiones del habón provocado por el procedimiento quirúrgico experimental.

\section{Espécimen 2}

Cantidad de producto aplicado: $0,4 \mathrm{cc}$

Mediciones obtenida

\section{Observador 1 Observador 2}

\begin{tabular}{lccc}
$\begin{array}{l}\text { Largo } \\
(\mathrm{mm})\end{array}$ & 12,27 & 12,50 & 12,38 \\
\hline $\begin{array}{l}\text { Ancho } \\
(\mathrm{mm})\end{array}$ & 7,39 & 7,97 & 7,68 \\
\hline $\begin{array}{l}\text { Alto } \\
(\mathrm{mm})\end{array}$ & 2,65 & 2,67 & 2,66 \\
\hline
\end{tabular}

\section{Espécimen 3}

Cantidad de producto aplicado:0,5 cc

Mediciones obtenidas Observador 1 Observador 2

\begin{tabular}{lccc}
$\begin{array}{l}\text { Largo } \\
(\mathbf{m m})\end{array}$ & 13,11 & 12,25 & 12,68 \\
\hline $\begin{array}{l}\text { Ancho } \\
(\mathrm{mm})\end{array}$ & 9,27 & 9,60 & 9,43 \\
\hline $\begin{array}{l}\text { Alto } \\
(\mathrm{mm})\end{array}$ & 3,26 & 3,09 & 3,17 \\
\hline
\end{tabular}

\section{Espécimen 4}

Cantidad de producto aplicado: $0,3 \mathrm{cc}$

Mediciones obtenidas:

Fue la primera disección que se realizó y no se midieron las dimensiones del producto en la zona infiltrada, al ser más cortante, buscando el confirmar que el gel no infiltraba las estructuras anatómicas.

\section{Espécimen 5}

Cantidad de producto aplicado: $0,2 \mathrm{cc}$

Mediciones obtenidas

\begin{tabular}{|c|c|c|c|}
\hline & Observador 1 & Observador 2 & $\bar{X}$ \\
\hline $\begin{array}{l}\text { Largo } \\
(\mathrm{mm})\end{array}$ & 11,36 & 12,11 & 11,73 \\
\hline $\begin{array}{l}\text { Ancho } \\
(\mathbf{m m})\end{array}$ & 10 & 10,21 & 10,10 \\
\hline \multicolumn{4}{|l|}{$\begin{array}{l}\text { Alto } \\
(\mathrm{mm})\end{array}$} \\
\hline $\begin{array}{l}\text { Apéndice } \\
(\mathrm{mm})\end{array}$ & 10,23 & 12,13 & 11,18 \\
\hline
\end{tabular}

\section{Espécimen 6}

Cantidad de producto aplicado: $0,4 \mathrm{cc}$

\begin{tabular}{|crrr}
\multicolumn{4}{c}{ Mediciones obtenidas } \\
\hline & Observador 1 & Observador 2 & $\bar{X}$ \\
\hline $\begin{array}{l}\text { Largo } \\
(\mathrm{mm})\end{array}$ & 7,79 & 7,27 & 7,54 \\
\hline $\begin{array}{l}\text { Ancho } \\
(\mathrm{mm})\end{array}$ & 6,66 & 6,50 & 6,58 \\
\hline $\begin{array}{l}\text { Alto } \\
(\mathbf{m m})\end{array}$ & 2,50 & 2,49 & 2,49 \\
\hline
\end{tabular}

\section{Espécimen 7}

Cantidad de producto aplicado: $0,3 \mathrm{cc}$

Mediciones obtenidas

\begin{tabular}{|c|c|c|c|}
\hline & Observador 1 & Observador 2 & $\bar{X}$ \\
\hline $\begin{array}{l}\text { Largo } \\
(\mathrm{mm})\end{array}$ & 10,37 & 9,98 & 10,17 \\
\hline $\begin{array}{l}\text { Ancho } \\
(\mathrm{mm})\end{array}$ & 7,80 & 8,98 & 8,29 \\
\hline $\begin{array}{l}\text { Alto } \\
(\mathrm{mm})\end{array}$ & 2,66 & 2,64 & 2,65 \\
\hline
\end{tabular}




\section{Espécimen 8}

Cantidad de producto aplicado: $0,5 \mathrm{cc}$

Mediciones obtenidas

\section{Observador 1 Observador $2 \quad \bar{X}$}

\begin{tabular}{lccc}
$\begin{array}{l}\text { Largo } \\
(\mathrm{mm})\end{array}$ & 8,99 & 9,55 & 9,27 \\
$\begin{array}{l}\text { Ancho } \\
(\mathrm{mm})\end{array}$ & 8,50 & 9,10 & 8,80 \\
\hline $\begin{array}{l}\text { Alto } \\
(\mathrm{mm})\end{array}$ & 3,25 & 3,11 & 3,13 \\
\hline
\end{tabular}

\section{Espécimen 9}

Cantidad de producto aplicado: $0,5 \mathrm{cc}$

Mediciones obtenidas

\section{Observador 1 Observador $2 \quad \bar{X}$}

\begin{tabular}{lccc} 
Largo & & 9,54 & 9,46 \\
\hline $\begin{array}{l}\text { Anm) } \\
(\mathrm{mm})\end{array}$ & 9,39 & 6,99 & 6,93 \\
\hline $\begin{array}{l}\text { Alto } \\
(\mathrm{mm})\end{array}$ & 6,88 & 2,08 & 4,41 \\
\hline
\end{tabular}

\section{Espécimen 10}

Cantidad de producto aplicado: $0,4 \mathrm{cc}$

Mediciones obtenidas

\begin{tabular}{|c|c|c|c|}
\hline & Observador 1 & Observador 2 & $\overline{\boldsymbol{X}}$ \\
\hline $\begin{array}{l}\text { Largo } \\
(\mathrm{mm})\end{array}$ & 10,31 & 10,92 & 10,66 \\
\hline $\begin{array}{l}\text { Ancho } \\
(\mathrm{mm})\end{array}$ & 8,78 & 8,89 & 8,83 \\
\hline $\begin{array}{l}\text { Alto } \\
(\mathbf{m m})\end{array}$ & & & \\
\hline
\end{tabular}

\section{Espécimen 11}

Cantidad de producto aplicado: $0,3 \mathrm{cc}$

Mediciones obtenidas

\begin{tabular}{|c|c|c|c|}
\hline & Observador 1 & Observador 2 & $\bar{X}$ \\
\hline $\begin{array}{l}\text { Largo } \\
(\mathrm{mm})\end{array}$ & 11,21 & 11,85 & 11,53 \\
\hline $\begin{array}{l}\text { Ancho } \\
(\mathrm{mm})\end{array}$ & 9,84 & 8,16 & 9,00 \\
\hline $\begin{array}{l}\text { Alto } \\
(\mathrm{mm})\end{array}$ & 3,60 & 3,00 & 3,30 \\
\hline
\end{tabular}

Datos obtenidos de las hojas de control de los especímenes

\section{Fecha de realización}

- 9 especímenes seleccionados el 20 de febrero de 2006

- 1 especímen el 7 de febrero de 2006

En el primer día de experimentación con los especimenes (n10), sólo se llevó a cabo la infiltración, así como la disección y medición posterior en un espécimen $(n=1)$. Los nueve restantes $(n=9)$, se realizaron en un único día, dos semanas más tarde.

\section{Espécimen}

Del total de los especimenes el $60 \%$ eran varones, mientras que el $40 \%$ restante eran hembras

\section{Edad de los especimenes}

La media de edad es de 82,5 años, siendo el rango de 66 a 91 años.

Distribución en porcentaje de las edades:

\begin{tabular}{ll}
\hline 1 caso de 66 años & $10 \%$ \\
\hline 2 casos de 78 años & $20 \%$ \\
\hline 1 caso de 80 años & $10 \%$ \\
\hline 2 casos de 84 años & $20 \%$ \\
\hline 1 caso de 85 años & $10 \%$ \\
\hline 1 caso de 88 años & $10 \%$ \\
\hline 2 casos de 91 años & $20 \%$ \\
\hline
\end{tabular}

\section{Localización topográfica de la lesión}

Todos los especímenes presentaban la lesión queratósica en el $5^{\circ}$ dedo.

Medio de detección de la exostosis

La detección de la exostosis se llevo a cabo mediante fluoroscopia en el 100\% de los casos.

\section{Presencia de exostosis}

En el 100\% de los casos la fluoroscopia mostró presencia de exostosis en la falange del $5^{\circ}$ dedo.

\section{Cantidad de producto infiltrado}

La cantidad de PAAG no fue la misma en todos los casos, según mostramos en cuatro grupos. 


\begin{tabular}{ll}
\hline $0.2 \mathrm{cc}$ en 1 caso & $10 \%$ \\
\hline $0.3 \mathrm{cc}$ en $3 \mathrm{casos}$ & $30 \%$ \\
\hline $0.4 \mathrm{cc}$ en $3 \mathrm{casos}$ & $30 \%$ \\
\hline $0.5 \mathrm{cc}$ en 3 casos & $30 \%$ \\
\hline
\end{tabular}

Tipo de aguja

Se utilizaron tres tipos de calibre diferentes.

\begin{tabular}{ccc}
\hline $25 / 5$ & 1 caso & $10 \%$ \\
\hline $25 / 6$ & 8 casos & $80 \%$ \\
\hline $38 / 8$ & 1 casos & $10 \%$ \\
\hline
\end{tabular}

\section{Forma de aplicación}

La forma de infiltración se realizó directamente sobre la exostosis en el $40 \%$ de los casos, mientras que en el $60 \%$ restante se llevó a cabo la infiltración en forma de abanico.

\section{Facilidad de infiltración del producto}

En el $80 \%$ de los casos la infiltración se produjo sin ningún tipo de resistencia ni dificultad, en el $20 \%$ restante se comprobó cierta dificultad a la hora de inyectar el producto.

\section{Observación de distribución del producto}

Para valorar la distribución del producto en el tejido, se realizó la disección de la zona en el $50 \%$ de los casos; siendo el $50 \%$ restante valorado mediante la sección longitudinal del dedo con una sierra Exakt.

Valoración de los resultados

\section{Distribución del producto}

La distribución del producto en el 100\% de los casos fue considerada como correcta por ambos observadores, no observándose invasión del PAAG en el tejido adyacente de la exostosis.

\section{Concentración del producto}

Se pudo comprobar que en los especímenes en los cuales se ha inyectado el PAAG, se logra correctamente un volumen de relleno de gel sobre la exóstosis que ocasiona un aislamiento de ésta de posibles presiones. Aún pasado un periodo de tiempo, y colocado el pie en diversas posiciones, no se ve modificada la posición, ni tampoco la localización del gel.

\section{Distribución en tejidos}

Se ha comprobado la no diseminación, ni infiltración del gel al resto de estructuras anatómicas del dedo. No invade ni el tejido dérmico, ni la cápsula articular, ni los componentes articulares (cartílago articular, tejidos óseo, periostio). Lo que significa que no hay ninguna indicación adversa para poder aplicar el producto en pacientes reales afectos de helomas digitales producidos por exostosis digitales, que provocan dolor, incapacidad y alteraciones biomecánicas.

La valoración de la técnica es positiva en el $100 \%$ de los casos, demostrando que el producto permanece in situ.

\section{DISCUSIÓN}

Aunque nuestra experiencia clínica nos demuestra la elevada prevalencia de exostosis interdigital en nuestra población, existe muy poca bibliografía que haga referencia a dicha patología, lo que dificulta la discusión de los resultados del presente estudio. La condición, en efecto, es más frecuente que lo escaso que las publicaciones sugiere, probablemente porque no se da a esta entidad clínica la suficiente importancia científica ${ }^{27}$.

Ello nos hace tomar como referencia principal nuestros propios estudios sobre la prevalencia y la eficacia de los tratamientos de esta patología, llevados a cabo en la Clínica Podológica de la Fundación Josep Finestres de la Universitat de Barcelona, en el que hasta el momento se han realizado dos tipos de tratamiento: ortesis de silicona la ortesis con el objetivo de mantener la separación y evitar las presiones sobre la zona dérmica, disminuyendo la intensidad de éstas; y tratamiento quirúrgico mediante técnica MIS con la finalidad de realizar un limado de la exostosis.

La causa de la formación del heloma, que constituye el principal signo clínico de la exostosis digital, es la presión que ejerce la exostosis sobre el tejido dérmico, agravada por la presión externa ejercida por el calzado en caso del $5^{\circ}$ dedo o el dedo lateral en el resto de los $\operatorname{dedos}^{4,27}$

Al estudiar los tratamientos realizados hasta la actualidad para esta patología, podemos 
constatar se suceden una serie de inconvenientes. En la terapia mediante la aplicación de ortesis de silicona, a pesar de conseguirse una mejoría importante del proceso, se debe plantear al paciente el cambio del tipo de calzado, por uno más amplio en la zona anterior, además de la necesaria utilización de un medio de sujeción de la ortesis como es la media o calcetín, con las consiguientes incomodidades. Además, en verano el paciente debe seguir llevando un calzado cerrado en la zona anterior, lo cual es mal tolerado.

El uso de ortesis de silicona presenta pues como complicación el rechazo de su uso por la incomodidad que causa en el paciente debido al contacto con el calzado. En nuestra casuística esta complicación alcanzó un 45,5\% de los casos, porcentaje que aumentó al $60 \%$ en la estación estival. Creemos que si logramos tratar a los pacientes mediante PAAG evitaremos esta complicación.

La terapia quirúrgica, si bien no implica un cambio del calzado, sí suele causar una baja laboral por un periodo mínimo de tiempo, además del traumatismo de la zona y al menos durante un periodo de al menos dos años, la no exposición de la zona cicatricial al sol ${ }^{10,11}$.

Referente al tratamiento con cirugía, observamos en nuestra casuística complicaciones inflamatorias ligeras en un $27,3 \%$ de los pacientes al final del periodo observacional.
Asimismo pudimos constatar la necesidad de que los pacientes solicitaran una incapacidad laboral transitoria a causa de este proceso, con una media de 15 días de baja laboral. Por el contrario, pensamos que con el tratamiento con PAAG, no será preciso la solicitud de baja laboral, exceptuando el reposo durante 12 horas que se debe realizar después de la infiltración.

Es importante resaltar las complicaciones que ocasiona esta patología al paciente, desde un dolor agudo, en algunos casos incapacitarte, hasta la disminución de su calidad de vida por los efectos biomecánicos de la presencia del heloma en la marcha. El hecho de que el heloma sea una entidad que pueda ser considerada menor, tanto por la escasa bibliografía existente, como por ser una lesión de la piel, no la exime de efectos secundarios indeseables, como son la infección, inflamación y ulceración ${ }^{14,13,10,11}$. Estos afectan incluso a la biomecánica general del esqueleto ${ }^{3}$. Pensamos que ninguno de estos síntomas se presentarán en los pacientes tratados con PAAG si la técnica es bien ejecutada, aunque será necesario su puesta en práctica in vivo para poder ratificar esta afirmación.

La valoración de la técnica es positiva en el $100 \%$ de los casos, demostrando que el producto permanece in situ en la localización prevista, lo que nos hace concluir que puede ser un tratamiento válido para la exostosis interdigital. 


\section{ANEXO}

Hoja de control en especímenes-pie

\section{HOJA DE CONTROL EN ESPECIMENES - PIE}

Ficha núm.:

Fecha de realización:

Identificación del espécimen:

Sexo: $\square$ Varón $\square$ Hembra

Edad:

Localización topográfica de la lesión:
$\square$ Interdigital:
$\square$ Dorso $5^{\circ}$ dedo:
$\square$ No hay exostosis

Medio de detección de la exostosis: $\quad \square$ Fluoroscopia $\square$ Radiografía Tiene exostosis: $\square$ Sí $\square$ No

Cantidad de producto aplicado: ............ml

Tipo de aguja: $\quad \square 25 / 5 \square 25 / 6 \square$ Otras

Forma de aplicación del producto: $\square$ Abanico $\square$ Otras.........

Facilidad de infiltración del producto

$\square$ Sin dificultad $\square$ Con dificultad:

Observación de distribución del producto

$\square$ Disección $\square$ Corte longitudinal

Distribución del producto

$\square$ Correcta $\square$ Alterada:

Valoración de la técnica

$\square$ Correcta $\square$ Negativa: 


\section{BIBLIOGRAFÍA}

1. Alonso J, Vázquez P, Fluvia J. Evaluación y tratamiento de los helomas interdigitales. Rev Esp Pod 1998;IX(1):33-38.

2. Breiting V, Aasted A, Jorgensen A, Opitz P, Rozetsky A. A study on patients treated with polyacrylamide hydrogel injection for facial corrections. Aesthetic Plast Surg 2004;28:45-53.

3. Butterworth R, Dockery G. Atlas a color y texto de cirugía del antepié. Madrid: Ortocen, 1992;p.85-91.

4. Canella P. Exostosis: development, evolution and relationship to malignant degeneration. Ital J Orthop Traumatol 1981;7(3):293-8.

5. Canfield AE, Doherty MJ, Ashton BA. Osteogenic potential of vascular pericytes. En: Davies JE ed. Bone Engineering. Toronto: Davies JE ed, 2000; p.143-51

6. Chevrot A. Diagnóstico por imagen de las afecciones del pie. Barcelona: Masson, 2000.

7. Christensen L, Breiting V, Janssen M, Vuust J, Hogdall E. Adverse reactions to injectable soft tissue permanent fillers. Aesthetic Plast Surg 2005; 29(1):34-48.

8. Christensen L, Breiting V, Vuust J, Hogdall E. Adverse reactions following injection with a permanent facial filler polyacrylamide hydrogel (Aquamid): cusesnand treatment. European Journal of Plastic Surgery 2006;28:464-471.

9. Christensen L. Normal and pathologic tissue reactions to soft tissue gel fillers. Dermatol Surg 2007;33(2):168-75.

10. Coughlin MJ. Mallet toes, hammer toes, claw toes and corns. Causes and treatment of lesser - toe deformities. Postgraduate Medicine 1984;75(5):191-198.

11. Coughlin MJ. Rheumatoid forefoot reconstruction. A long-term follow-up study. The Journal Of Bone And Joint Surgery. American Volume [serial online] 2000;82 (3):322-341. [consultado 25-07-2011] Disponible en: MEDLINE, Ipswich, MA.

12. Dahlin DC. Tumores óseos. $2^{\mathrm{a}}$ edición. Barcelona: Toray, 1980;p.14-23.

13. Dalton E, Louis AJ, Green D. Lesser ray deformities. In: Banks, AS. et.al. - Comprehensive textbook of Foot and Ankle Surgery. $3^{\text {a }}$ Ed. Philadelphia: Lippincott Williams \& Wilkins, 2001; p.253 - 304.

14. Day RD, Reyzelman AM, Harkless LB. Evaluation and management of the interdigital corn: a literature review. Clinic Podiatr Med Surg 1996;13(2):201-6.

15. De Palma L, Gigante A, Specchia N. Subungual exostosis of the foot. Foot \& Ankle Int 1996;17(12):758763.

16. DiGiovanni, C. Core Knowledge in orthopaedics: foot and ankle. Ed. Saunders, 2007.

17. Evision GE, Price CHG. Subungueal exostosis. Br J Radiol 1966;39 (462):451-455.

18. Frank ED. Guía de bolsillo de radiología de Merril. Madrid: Elsevier, 2008.

19. Greenfiel GB. Radiology of bone diseases. 5 ed. Philadelphia:JB Lippincott, 1990.

20. Hudson TM, Springfield DS, Spanier SS, Enneking WF, Hamlin DJ. Benign exostoses and exostotic chondrosarcomas: evaluation of cartilage thickness by CT. Radiology 1984 Sep; 152 (3): 595-9.

21. Landon CG, Johnson KA, Dahlin DC. Subungual exostosis. J. Bone Joint Surg 1979;61 A:256-259.

22. Li Y, Yue T, Han Y. Subungueal exostosis. Chin Med Sci J 1991;6:169-171.

23. Lichtenstein L. Bone tumors. St Louis, MO, USA: CV Mosby Co,1972: p.17-28

24. Mathewson MH. Subungueal exostose of the fingers: are they really uncommon? Br J Dermatol 1978;98:187-189.

25. Murphey MD, Choi JJ, Krandsdorf MJ, Flemming DJ, Ganon FH. Imaging of osteochondroma: variants and complications with radiologic-pathologic correlation. Radiographics 2000;20(5):1407-34.

26. Nuñez-Samper M, Llanos LF. Biomecánica, medicina y cirugía del pie. Barcelona: Masson, 2000; p.256-263.

27. Ortega del Olmo RM, Sanchez HG, Naranjo SR. Veintitrés observaciones de exostosis. Med Cut I L A 1988; XVI:11-14.

28. Prado M (de), Ripoll P, Golanó P. Cirugía Percutánea del Pie. Barcelona: Masson, 2004; p.221-233.

29. Shelley DE, Shelley WB. Exploratory nail plate removal as a diagnostic aid in painful subungueal tumor: glomus tumor, neurofibroma and squamous cell carcinoma. Cutis 1986;39:310-312.

30. Tuan-anh Le A. Rhinoplasty using injectable polyacrylamide gel - a patient study. Australian journal of cosmetic surgery 2005;1(1).

31. Viladot A, Viladot R. 20 lecciones sobre patología del pie. Barcelona: Mayo ediciones, 2009.

32. von Buelow S, von Heimburg D, Pallua N. Efficacy and Safety of Polyacrylamide Hydrogel for Facial Soft-Tissue Augmentation. Plast. Reconstr. Surg 2005;116:1137-1146. 
33. von Buelow S, Pallua N. Efficacy and Safety of Polyacrylamide Hydrogel for Facial Soft-Tissue Augmentation in a 2-Year Follow-Up: A Prospective Multicenter Study for Evaluation of Safety and Aesthetic Results in 101 Patients. Plast Reconstr Surg 2006;118: 85S.

34. Yafee HS. Peculiar nail dystrophy caused by an enchondroma. Arch Dermatol 1965; 91: 361-362.

35. Zarini E, Supino R, Pratesi G, Laccabue D, Tortoreto M, Scanziani E, et al. Biocompatibility and tissue interactions of a new filler material for medical use. Plast Reconstr Surg 2004; 114: 934-942. 